\title{
Vaccines: An Armament in Combating Antimicrobial Resistance
}

\author{
Maria Khan \\ Department of Microbiology, Rehman Medical Institute, Peshawar, Pakistan
}

Antimicrobial resistance (AMR) is a worldwide risk to healthcare, consequently increasing the healthcare costs as well as mortality and intimidating prospective medical procedures. Customarily, AMR is addressed through antibiotic stewardship, infection prevention and control precautions, promotion of antibiotic research, and development, agricultural/livestock use restriction, antibiotic cycling, antibiotic mixing, as well as prompt and accurate diagnostics. ${ }^{1}$ At the same time, multifacetedstrategies takeinto accounttheincreasing awareness, safeguarding practices related to environmental protection (feed production, food and waste management), surveillance improvement and research, human and animal vaccination programmes, better sanitation and optimising the antibiotic use. ${ }^{2}$ World Health Organization (WHO) listed AMR, amongst ten substantial threats to global health, yet it is likely that by 2030 , economic impact of AMR will be comparable to global climate change. ${ }^{3}$ Apart from this, emerging economies have predicted $60 \%$ increase in cost on antibiotic from 2020 to 2030. ${ }^{4}$ By 2050, AMR associated estimated cost for global economy will increase to USD $100,000 .^{5}$ The World Bank report on antibiotics use peryear globally on agriculture and livestock is appraised to be $63,000-240,000$ metric tons. ${ }^{6}$ Overall, AMR has impact on global trade, gross domestic product, livestock raising, and healthcare budget, due to escalating morbidity. ${ }^{1}$

In USA, $80 \%$ of antibiotics are used in animals, mostly to enhance yield and quality of meat. Whereas, in developing countries antibiotics are offered without a prescription, which lead to unconstrained and indiscriminate use in population. ${ }^{7}$

Generally, the annual number of estimated deaths globally, due to $A M R$, is 700,000 , including 230,000 deaths attributable to multidrug resistant (MDR) tuberculosis. ${ }^{8}$ Usually, the inability to promptly identify pathogen at point of care leads to empirical treatment, eventually imposing pressure on microorganisms besides targeted pathogen, leading to bystander selection.

Correspondence to: Dr. Maria Khan, Department of Microbiology, Rehman Medical Institute, Peshawar, Pakistan

E-mail: kmaria22@hotmail.com

Received: February 17, 2021; Revised: March 01, 2021;

Accepted: March 16, 2021

DOI: https://doi.org/10.29271/jcpsp.2021.03.249
To elucidate, Alexander Fleming in 1946 predicted the overall burden with this renowned verdict, "There is probably no chemotherapeutic drug to which, in suitable circumstances the bacteria cannot react by in some way acquiring fastness [resistance]". Consequently, penicillin was commercialised in 1943 and resistance development was noticed by 1948 in staphylococcusaureus. ${ }^{9}$

Why are vaccines considered to be an extreme tool against AMR? Firstly, resistance development is a mere issue in vaccination because there are no spontaneous mutations or the mobile genetic element acquirement by horizontal gene transfer. Due to prophylactic use of vaccines, there is lesser likelihood of resistance-conferring mutations that can appear or proliferate smaller pathogen population. It attacks microbes in various ways, requiring multiple mutations to confer resistance; in contrast, antibiotics have single target. In addition, vaccines induce T cell responses or host-specific antibody; and sometimes a combination of both. Secondly, its golden era of vaccines, where resistance selection is making the antibiotics obsolete. In contrast, there is a dire need of continual pipeline of novel antibiotics for effective treatment. Hardly any new antibiotics have been licensed in the course of the last 40 years. Furthermore, no antibiotic employing a new mechanism against gram-negative bacteria has been announced in current timeframe. ${ }^{1}$ During the last century in USA, the statistics on licensed products shows declension of antibiotic era and vaccine escalation. ${ }^{10}$ Thirdly, by decreasing global disease incidence covering both infections caused due to viruses and susceptible pathogens, decreases both inappropriate and appropriate antimicrobial usage. ${ }^{11}$ In ambulatory care in the USA, a third of antibiotic prescriptions are for viral infections. Another reason to use vaccines for combating AMR is to preserve the microbiome; to reduce detrimental impact on overall health, particularly in children; to reduce alteration in the immune system's development and negative effects on nutritional status. Markedly, antibiotics alter the microbiome composition, through bystander selection, which indirectly generate a pool of host-specific resistant genes. ${ }^{12}$

Firstly, vaccines can aid in averting AMR by inhibiting disease incidence and microbial proliferation. Theoretically, there are almost billions of copies of the pathogen, which directly increase mutation probability and confer antibiotic resistance. ${ }^{13}$ Likewise, it restricts pathogen ability to institute a foothold in the host by inducing immune responses to multiple 
targets (e.g., multiple epitopes on each antigen). Therefore, bacteria would need to undergo multiple mutations to escape the immunity induced by vaccination. ${ }^{14}$ Increasing vaccination coverage also offers an indirect herd protection. Clinicians often prescribe antibiotics empirically (e.g. suspected typhoid fever) to avoid complications mortality, for infections requiring confirmation through culture and antibiotic susceptibility, mostly due to expensive culture and susceptibility tests or lack of testing facility. ${ }^{15}$ Therefore, downsizing these medicaments through vaccination will have primary control on AMR by preventing antimicrobial misuse. Currently, worldwide cases by Salmonella enterica serotype typhi sores to approximately 20 million cases and greater than 150,000 deaths. However, data regarding exact incidence of typhoid fever (AST confirmed) is considerably lower, with 3 in 25 cases treated for each true case, results in over 50 million avoidable antibiotic prescriptions every year. ${ }^{16}$ At this time, viral febrile illness mimicking symptoms of typhoid fever are the main drivers for antibiotic misuse across South Asia. For this purpose, WHO recommends the prioritisation of typhoid vaccination in high encumbering nations; and in particular, with heightened AMR prevalence. ${ }^{15}$ Equally, influenza vaccine can scale down incorrect prescriptions for symptoms of upper respiratory tract illness. Throughout winter months, there is $10-30 \%$ additional antimicrobial usage owing to influenza; and through vaccination, acute otitis media cases can be reduced by a third. ${ }^{17}$ During the year 2000, universal influenza vaccination implementation in Ontario, lead to reported $64 \%$ decline in prescriptions leading to 144,000 antimicrobial prescriptions avoidance per year. ${ }^{18}$ As revealed by a meta-analysis of 96 studies, influenza vaccination cut down days of antibiotic utilisation amongst healthy individuals (28.1\% reduction) and anti-microbial regulation in pediatric age group ( 6 monthstill 14 years). ${ }^{19}$

Next, by subsiding antibiotic utilisation due to sporadic infections from Streptococcus pneumoniae (>90 serotypes identified) being the leading cause of childhood hospitalisation, otitis media, bronchitis, antibiotic use and mortality from severe life-threatening pneumonia, invasive pneumococcal diseases (IPD, meningitis and bacteremia). In year 2000, USA, started pneumococcal conjugate vaccine 7 (PCV-7: 4, 6B, 9V, $14,18 \mathrm{C}, 19 \mathrm{~F}, 23 \mathrm{~F})$ routine childhood vaccination; and by 2007 , IPD cases due to all serotypes had waned by $76 \%$ in children below 5 years for $100 \%$ vaccine serotypes, $37 \%$ case reduction in people over 65 years (for $92 \%$ vaccine serotypes). ${ }^{20}$ Afterwards, antibiotic prescriptions dropped by $35 \%$ in vaccinated pediatric age group and a prospective decrease of 1.4 million antibiotic prescriptions each year in Northern California. ${ }^{21}$

In 2010, infections due to serotype replacement (non-vaccine serotypes inclination) were prevalent in USA, for that PCV 13 (six additional cserotypes not constituted in PCV-7) was introduced. In Africa, USA and Europe, pneumococcal conjugate vaccine reduced resistant invasive pneumococcal disease as well as antibiotic use. ${ }^{21}$ Eventually, Pakistan was the foremost country in South Asian region to offer PCV-10 vaccine in phased manner, started October 2012 in routine immunisation program. (GoP \& GAVI) ${ }^{22}$ Clearly, acute otitis media (AOM) is another most common cause of antibiotic prescriptions in the USA, pneumococcal polysaccharide conjugate vaccines induced direct protection of infants to AOM and developed herd immunity in adult age group. ${ }^{23}$ In the same way, PCV7, PCV13 and pneumococcal haemophilus influenzae protein D conjugate vaccine (PHiD-CV), reduced prevalence of pneumococci resistant to penicillin among invasive strains and IPD cases. ${ }^{24} \mathrm{By}$ decreasing AOM and pneumonia, widespread antibiotic utilisation ratio is anticipated to drop and eventually decrease AMR rates. During 2004, resistant strains prevention from occurring/spreading, PCV-7 serotype infection declined by $87 \%$ in the USA; and by 2008, PCV-7 reduced IPD due to penicillin-non-susceptible by $64 \%$ in children below 5 years, and $45 \%$ in adults over 65 yearsin USA. ${ }^{1}$

In the past, 1970, increasing resistance to $\beta$ - lactam in $H$. influenzae type $\mathrm{b}(\mathrm{Hib})$ that was responsible for severe lifethreatening childhood infections. As vaccines are rarely designed to target resistant pathogens directly; but with introduction of Hib vaccination in 1988, $\beta$-lactamase-positive Hib strains infection steadily declined. Until 2006, Hib vaccination was not largely implemented in developing countries, as it was unequivocally endorsed by the WHO. ${ }^{25}$ Furthermore, vaccines can reduce infection due to resistant strains among inter-related species, as noted in retrospective case-control study between 2004 and 2008, where one million individuals were inoculated outer membrane vesicles based vaccine (MeNZB) against meningococcus B outbreak in New Zealand, which leads to $31 \%$ reduction of gonorrhea cases. ${ }^{26}$ Evident from the fact that $N$. meningitides and N. gonorrhoeae has $80-90 \%$ nucleotide homology at the gene level. Advance research is necessary to assess the efficacy of MenB-4C vaccine in inhibiting gonococcal infections. Initially, infection prevention through vaccines decrease the antibiotics (and antivirals) utilisation for co-infections or superinfections treatment.

In a Swedish study, 35\% of cases hospitalisation due to adult community acquired pneumonia were mixed infections, majority caused by $S$. pneumoniae in addition to respiratory viruses. ${ }^{27}$ Another multicentre study, $65 \%$ of adults with laboratory-confirmed influenza revealed secondary bacterial infections, predominantly S. pneumoniae and S. aureus infections. ${ }^{28}$ These cases lead to increased morbidity, mortality as well as antibiotic usage that could have been prevented by PCV. Individuals with influenza are often more susceptible to secondary bacterial infections; therefore, vaccines can prevent these complications. $^{29}$

Future research is going to develop vaccine against some methicillin Resistant Staphylococcus aureus genotypes as well as treatment for drug-susceptible genotypes in reducing hospital outbreaks. ${ }^{30}$ Moreover, hindrance to influenza vaccination is mostly due to decreased perception of disease severity and doubts regarding safety and effectiveness of immunisation. Using recombinant DNA, glycoconjugation and reverse vacci- 
nology accord for optimal vaccine antigens designing. Likewise, a novel glycoconjugate vaccine for Klebsiella pneumoniae and Psuedomonas aeruginosa infections is in preclinical development. Other approaches to enhance vaccine efficacy are growing serotype coverage, vaccine-antibiotic combined strategy, vaccine adjuvants, structural vaccinology, bio-conjugates, genetically modified outer membrane vesicles and therapeutic monoclonal antibodies (mAbs). ${ }^{13}$

In conclusion, the need is that resources for combating AMR should be dispensed among the competing options with strategy development for the best implementation of an integrated AMR strategy. Governing the prime apportionment of funds to address AMR is needed. Collective efforts in antibiotics field, novel tools for vaccine research and monoclonal antibodies will deliver affordable resolutions to confront AMR.

\section{REFERENCES}

1. Buchy $P$, Ascioglu S, Buisson $Y$, Datta $S$, Nissen M, Tambyah PA, et al. Impact of vaccines on antimicrobial resistance. Int J Infect Dis 2020; 90:188-96. doi: 10.1016/j.ijid.2019. 10.005 .

2. Geering WA, Roeder PL, Obi TU, Rweyemamu MM. Manual on the preparation of national animal disease emergency preparedness plans. Rome, Italy: FAO; 1999.

3. Jasovský D, Littmann J, Zorzet A, Cars O. Antimicrobial resistance-a threat to the world's sustainable development. Ups J Med Sci 2016; 121(3):159-64. doi: 10.1080/ 03009734.2016.1195900.

4. Dadgostar P. Antimicrobial resistance: implications and costs. Infect Drug Resist 2019; 12: 3903-10. doi: 10.2147/ IDR.S234610.

5. National Academies of Sciences, Engineering, and Medicine. The Cost Dimensions of Antimicrobial Resistance. In Understanding the Economics of Microbial Threats: Proceedings of a Workshop 2018 Oct 15. National Academies Press (US).

6. World Bank. Drug-resistant infections: A threat to our economic future. World Bank; 2017 Mar.

7. Torres NF, Solomon VP, Middleton LE. Patterns of selfmedication with antibiotics in Maputo City: A qualitative study. Antimicrob Resist Infect Control 2019; 8:161. doi: 10.1186/s13756-019-0618-z. eCollection 2019.

8. World Health Organization. Antibacterial agents in clinical development: An analysis of the antibacterial clinical development pipeline, including tuberculosis. 2017.

9. Rosini R, Nicchi S, Pizza M, Rappuoli R. Vaccines Against Antimicrobial Resistance. Front Immunol 2020; 11:1048. doi: 10.3389/fimmu.2020.01048.

10. Bloom DE, Black S, Salisbury D, Rappuoli R. Antimicrobial resistance and the role of vaccines. Proc Natl Acad Sci USA 2018; 115(51):12868-71. doi: 10.1073/pnas.1717157115.

11. Holmes KK, Bertozzi S, Bloom BR, Jha P, Gelband H, DeMaria LM, et al. Major infectious diseases: Key Messages from Disease Control Priorities, Third Edition. In: Major Infectious Diseases. 3rd edition. Washington (DC): The International Bank for Reconstruction and Development /
The World Bank; 2017 Nov 3. Chapter 1. DOI: 10.1596/9781-4648-0524-0_ch1

12. Relman DA, Lipsitch M. Microbiome as a tool and a target in the effort to address antimicrobial resistance. Proc Natl Acad Sci USA 2018; 115(51):12902-10. doi: 10.1073/pnas. 1717163115.

13. Micoli F, Bagnoli F, Rappuoli R, Serruto D. The role of vaccines in combatting antimicrobial resistance. Nat Rev Microbiol 2021; 1-16. doi: 10.1038/s41579-020-00506-3.

14. Lipsitch $M$, Siber GR. How can vaccines contribute to solving the antimicrobial resistance problem?. mBio 2016; 7(3):e00428-16. doi: 10.1128/mBio.00428-16.

15. Rasheed MK, Hasan SS, Ahmed SI. Extensively drugresistant typhoid fever in Pakistan. Lancet Infect Dis 2019; 19(3):242-3. doi: 10.1016/S1473-3099(19)30051-9.

16. Awol RN, Reda DY, Gidebo DD. Prevalence of Salmonella enterica serovar Typhi infection, its associated factors and antimicrobial susceptibility patterns among febrile patients at Adare general hospital, Hawassa, southern Ethiopia. BMC Infect Dis 2021; 21(1):30. doi: 10.1186/s12879-02005726-9.

17. Norhayati MN, Ho J, Azman MY. Influenza vaccines for preventing acute otitis media in infants and children. Cochrane Database Syst Rev 2017; 10(10):CD010089. doi: 10.1002/14651858.CD010089.pub3.

18. Kwong JC, Maaten S, Upshur RE, Patrick DM, Marra F. The effect of universal influenza immunization on antibiotic prescriptions: an ecological study. Clin Infect Dis 2009; 49(5):750-6. doi: 10.1086/605087.

19. Buckley BS, Henschke N, Bergman H, Skidmore B, Klemm EJ, Villanueva $G$, et al. Impact of vaccination on antibiotic usage: a systematic review and meta-analysis. Clin Microbiol Infect 2019; 25(10):1213-25. doi: 10.1016/ j.cmi.2019.06.030.

20. World Health Organization. Pneumococcal conjugate vaccine for childhood immunization - WHO position paper. Weekly Epidemiological Record = Relevé épidémiologique hebdomadaire. 2007; 82(12):93-104.

21. Klugman KP, Black S. Impact of existing vaccines in reducing antibiotic resistance: Primary and secondary effects. Proc Natl Acad Sci USA 2018; 115(51):12896-901.

22. Ali A, Husain S, Riaz A, Khawar H. Status of introduction of pneumococcal conjugate vaccine in Pakistan. Pediatr Infect Dis 2016; 8(2):64-6.

23. Qureishi A, Lee Y, Belfield K, Birchall JP, Daniel M. Update on otitis media-prevention and treatment. Infect Drug Resist 2014; 7:15-24. doi: 10.2147/IDR.S39637.

24. Tan TQ. Pediatric invasive pneumococcal disease in the United States in the era of pneumococcal conjugate vaccines. Clin Microbiol Rev 2012; 25(3):409-19. doi: 10.1128/CMR.00018-12.

25. Tristram S, Jacobs MR, Appelbaum PC. Antimicrobial resistance in Haemophilus influenzae. Clin Microbiol Rev 2007; 20(2):368-89. doi: 10.1128/CMR.00040-06.

26. Holst J, Oster P, Arnold R, Tatley M, Næss L, Aaberge I, et al. Vaccines against meningococcal serogroup $B$ disease containing outer membrane vesicles (OMV): lessons from past programs and implications for the future. Hum Vaccin 
Immunother 2013; 9(6):1241-53. doi: 10.4161/hv.24129.

27. Johansson N, Kalin M, Tiveljung-Lindell A, Giske CG, Hedlund J. Etiology of community-acquired pneumonia: increased microbiological yield with new diagnostic methods. Clin Infect Dis 2010; 50(2):202-9. doi: 10.1086/ 648678.

28. Morris DE, Cleary DW, Clarke SC. Secondary bacterial infections associated with influenza pandemics. Front
Microbiol 2017; 8:1041. doi: 10.3389/fmicb.2017.01041.

29. Manohar P, Loh B, Nachimuthu R, Hua X, Welburn SC, Leptihn S. Secondary bacterial infections in patients with viral pneumonia. Front Med (Lausanne) 2020; 7:420. doi: 10.3389/fmed.2020.00420.

30. Rasmussen RV, Fowler Jr VG, Skov R, Bruun NE. Future challenges and treatment of Staphylococcus aureus bacteremia with emphasis on MRSA. Future Microbiol 2011; 6(1):43-56. doi: 10.2217/fmb.10.155. 